\title{
Gradient Non-Linearity Correction of MR Images for Functional Radiosurgery
}

\author{
Tom S. Lee*†, Keith E. Schubert* \\ California State University, San Bernardino \\ Department of Computer Science \\ tom@r2labs.org, schubert@csci.csusb.edu
}

\author{
Reinhard W. Schulte ${ }^{\dagger}$ \\ Loma Linda University Medical Center \\ Department of Radiation Medicine \\ rschulte@dominion.llumc.edu
}

\begin{abstract}
This paper discusses the correction of MR images to submillimeter accuracy needed for functional radiosurgery. MR images experience non-linear distortion due to the magnetic field, which becomes more of a problem for newer machines with larger bores and stronger magnetic fields. This paper models the distortion correction parameters using a spherical harmonics basis, which avoids the need to invert the function to correct the image. The coefficients appear linearly for the spherical harmonics so they are solved for in each dimension by least squares techniques for an MR image of a phantom and the measurements of the phantom. Practical considerations in the design are also covered.
\end{abstract}

Keywords: Magnetic resonance imaging, Biomedical image processing, Nonlinear distortion

\section{Introduction}

Functional radiosurgery is a non-invasive treatment technique for creating small lesions in diseased areas of the brain to treat symptoms of functional disorders such as trigeminal neuralgia and Parkinson's disease [3]. A system for functional proton radiosurgery based on the sharp penumbra of high-energy proton beams $(250 \mathrm{MeV})$ is currently being developed at Loma Linda University Medical Center, Loma Linda, CA. Successful application of this method requires a high degree of geometric accuracy in localizing the intracranial target with high-resolution MRI. Nonlinearity of the magnetic gradient fields, the most prominent source of geometric distortion in modern MR scanners, can negatively influence the accuracy of target localization [1]. Several MRI distortion correction methods have been described in the literature, which are usually based on scanning a phantom with accurately known reference structures $[2,4,6,7]$. These are detected by a

\footnotetext{
* Supported by the NSF under award CISE 9810708.

${ }^{\dagger}$ Supported by the Henry L. Guenther Foundation.
}

specifically designed software, and a distortion correction model for the distortion between the ideal and the detected structures is assumed for which the model parameters are estimated, using a least squares fitting procedure.

The implementation of a software-based gradient nonlinearity distortion correction is indispensable for the newlyproposed functional proton radiosurgery system. The goal of the new system is to target specific brain areas with high doses of proton beams with submillimeter accuracy. Localizing the anatomical target with an MRI-based fiducial system requires correction of gradient nonlinearity distortions inherent in the scanner images. Modern MR scanners are particularly prone to such distortions due to wider bores and stronger gradient fields. The gradient nonlinearity correction described in this work is based on a high-resolution 3D MR scan of a cube phantom. Using a least-square fitting procedure, correction parameters are found that convert the geometrically warped planes of the cube into the ideal planes.

This implementation is based on the previous work of Langlois, et al [4]. Our correction model and its software implementation, however, differs in various key aspects from that work. In this report, we describe our method and present initial results.

\section{Image Preprocessing}

Creating a functional proton radiosurgery system accurate to within less than a millimeter requires a robust software acquisition and verification system. The software system will perform several tasks, for the purpose of enabling the proton radiosurgery system to accurately and safely hit a designated target. These tasks include data quality assurance, ideal surface calculation, and distortion correction.

The first stage of the software system will ensure that the data obtained from the MRI scanner is suitable for further calculation. This initial stage of the image processing is described in [5] and so will only be briefly reviewed here. An oil-filled cubic phantom is scanned with axial, coronal and sagittal sequences. Scanning the phantom provides a 


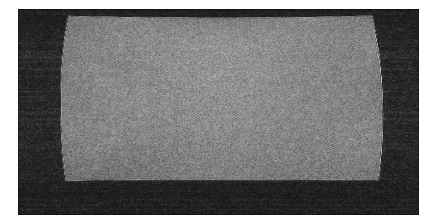

(a)

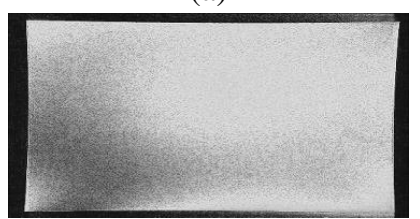

(c)

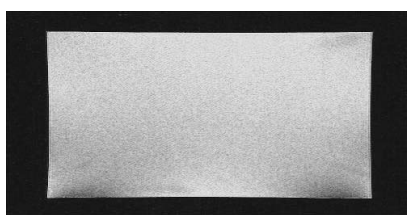

(b)

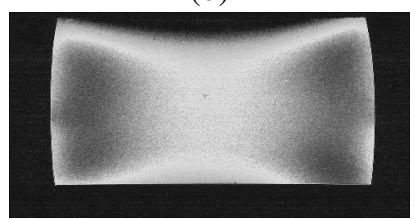

(d)
Figure 1. MR scanned images showing: (a-b) good slices, (c) slice with an oil leak in the lower right hand corner, (d) slice with only a partial view of the phantom. Slice (c) is corrected, while slice (d) is removed before analysis.

series of images, see Figure 1. Canny edge detection is then performed on the acquired images, in order to provide a representative data model of the phantom. The detected edges provide a two-dimensional representation of the phantom, which will be used to perform the gradient nonlinearity distortion correction in subsequent steps.

Prior to calculating the parameters of the distortion correction model, the data undergoes a series of image processing and quality checks. The quality checks have three main goals:

1. eliminate as much image noise as possible,

2. remove extraneous and unwanted features in each MR image, and

3. ensure the data is as accurate as possible for future calculations.

The ultimate accuracy of the correction will depend on the quality of the data produced after these data processing steps. Each quality check is executed as an individual module during this initial data processing stage. The data collected here represents the distorted surfaces of the phantom.

The data quality assurance steps include:

1. Determining useful images (images with oil leaks and partial views can not be used, see Figure 1).

2. Removing unwanted phantom features (i.e., the drain plug which should not be used).

3. Edge verification (checks that no large holes or noisy features exist on the edges).
4. Corner verification (checks that no large air bubble or noisy feature has corrupted the corner).

5. Air bubble removal (tiny air bubbles exist in any oil filled phantom and will introduce errors if not removed from the images).

6. Hole repair (the Canny edge detector can leave small holes which must be filled).

7. Verification of phantom centering inside MRI scanner. Misalignment of more than $1 \mathrm{~mm}$ would produce faulty results.

8. Calculation of phantom rotational tilt inside MRI scanner. Significant rotation (more than 1 degree) would produce faulty results.

Execution of these steps will ensure that the data is as clean as possible. Failure to remove or correct for extraneous image features would adversely affect the accuracy of all subsequent data processing steps, including the final distortion correction.

\section{Midplane Calculation}

In the second stage, the processed distorted data is used to model the undistorted surfaces of the phantom. The final gradient nonlinear distortion correction method is based on a set of calculated "ideal" planes. These planes represent the appearance of the faces of the phantom, without subjection to distortion. Although these planes are estimations of the actual faces, they can be calculated to a high degree of accuracy. The errors that result from these calculations are quite minute, and do not adversely affect the final calculations.

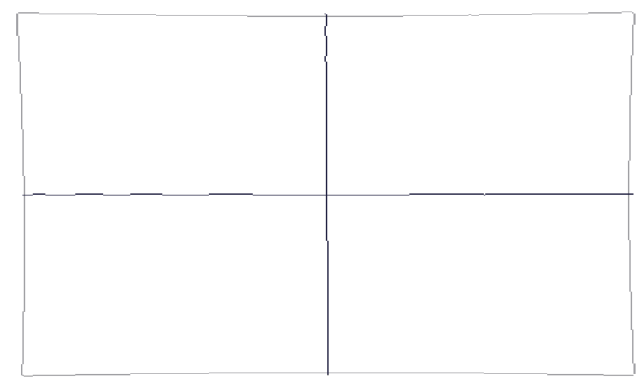

Figure 2. The upper and lower edge points are averaged to form a midline, as are the left and right edges. A plane is then fit to all the slices' midlines. 
Each pair of opposite edge points in the edge images is used to calculate a midpoint. The result of midpoint calculation along an entire edge produces a midline, see Figure 2. The algorithm excludes those points in the lists that do not have opposite neighbors, to ensure that the midpoint calculations generate valid results. The edge lengths can vary depending on the appearance of the distortion in the image, so it is acceptable to have opposite edges of slightly different lengths; the algorithm removes unpaired edge points to obtain edges of equal length.

Two midlines are calculated for each edge image: one horizontal, and one vertical. Due to the symmetry of gradient distortion, these midlines are nearly straight, and provide a suitable representation of the orientation of the undistorted edges in each image, disregarding the positioning of the edges. The midpoints from the entire set of images serve as the data that models the ideal midplanes with typical studies having around 30,000 midpoints. This midplane represents the ideal shape, size, and orientation of the particular surface on the phantom.

A plane equation is calculated that best fits the midplane data set in the least squares sense. The equation of a plane is of the form

$$
N^{T} x=d,
$$

where $N$ is the normal vector to the plane, $d$ is distance of the plane from the origin, and $x$ is the set of vectors satisfying the plane equation.

For example, fitting a plane to the top and bottom edges of the images in an axial study results in a plane oriented in XZ. Therefore, the plane can be expressed by

$$
\begin{aligned}
y & =-\frac{N_{1}}{N_{2}} x-\frac{N_{3}}{N_{2}} z+\frac{d}{N_{2}} \\
& =C_{x} x+C_{z} z+C_{c} \\
& =\left[\begin{array}{lll}
x & z & 1
\end{array}\right]\left[\begin{array}{l}
C_{x} \\
C_{z} \\
C_{c}
\end{array}\right]
\end{aligned}
$$

Thus based on the determined orientation, the algorithm organizes the data into one large matrix of dimension $n \times 3$, and one large array of dimension $n$, where $n$ is the number of midpoints. The $n \times 3$ matrix is the design matrix, denoted by $A$, and the $n$-element array is the observation vector, denoted by $b$. The resulting problem is

$$
A \chi=b
$$

where $\chi$ is a vector of the plane coefficients. In the example mentioned above, $b$ would contain y-data, and $A$ contains data from the other two dimensions (x-data and z-data), plus a column of ones. The column of ones corresponds to the offset term in the rearranged plane equation, $\frac{d}{N_{2}}$. For $n$ data points this leads to the linear system

$$
\left[\begin{array}{c}
y_{0} \\
y_{1} \\
\vdots \\
y_{n-1}
\end{array}\right]=\left[\begin{array}{ccc}
x_{0} & z_{0} & 1 \\
x_{1} & z_{1} & 1 \\
\vdots & \vdots & \vdots \\
x_{n-1} & z_{n-1} & 1
\end{array}\right]\left[\begin{array}{c}
C_{x} \\
C_{z} \\
C_{c}
\end{array}\right]
$$

Equation 6 can then be solved for the midplane coefficients using a technique like QR or SVD.

\section{Ideal Planes}

The midplanes describe the correct orientation of the ideal phantom planes, but they are not in the proper location. The basic idea of this section is to shift the midplanes along its normal vector by one half the physical dimensions of the phantom to their correct location in 3-dimensional space. The three phantom dimensions were derived from dimensional inspection and stored prior to algorithm execution. The result of this step is a set of six ideal planes, one for each of the six square faces of the phantom. These ideal planes describe how the faces of the phantom would theoretically appear in the absence of gradient nonlinearity distortions, see Figure 3. Note that these ideal planes are close but not identical to fitting planes to the original sides, as the averaging forces the opposing faces to be parallel and the shifting forces the faces to be at the right distance from each other and the midplane.

To shift the midplane in terms of the coefficients of the midplane, only the offset term is changed as the other coefficients must remain the same in order to maintain the midplane's original orientation. To calculate the shift, consider the plane equation, Equation 1, in terms of the coefficients defined in Equation 6 of the example in the last section.

$$
\begin{aligned}
N^{T} x & =C_{c} \\
N & =\left[\begin{array}{c}
-C_{x} \\
1 \\
-C_{z}
\end{array}\right]
\end{aligned}
$$

The normal vector must be normalized in order to prevent any unnecessary weighting in the final result. Normalization of $N$ produces the unit normal vector, $\bar{N}$,

$$
\begin{aligned}
& \bar{N}=\frac{N}{\|N\|} \\
& =\left[\begin{array}{c}
\frac{-C_{x}}{\sqrt{C_{x}^{2}+1+C_{z}^{2}}} \\
\frac{1}{\sqrt{C_{x}^{2}+1+C_{z}^{2}}} \\
\frac{-C_{z}}{\sqrt{C_{x}^{2}+1+C_{z}^{2}}}
\end{array}\right] .
\end{aligned}
$$

Consider a point,

$$
p=\left[\begin{array}{c}
0 \\
C_{c} \\
0
\end{array}\right]
$$



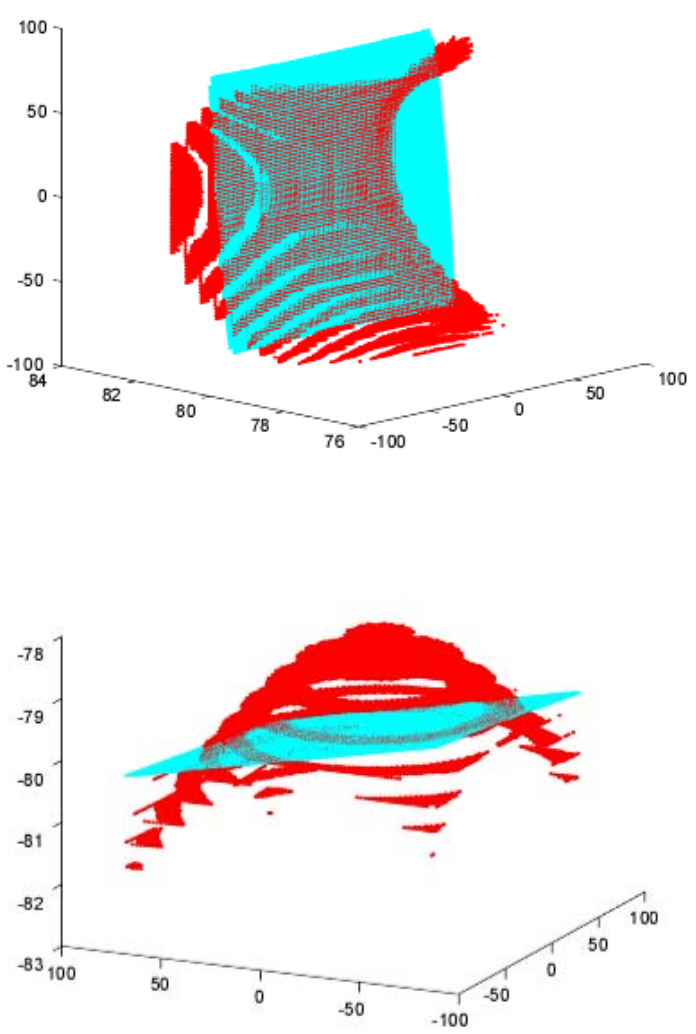

Figure 3. Distorted faces produced by MR scanner, and the ideal planes describing the phantom that produced them. The axis dimensions are pixels, and note the curvature is exaggerated so that it is visible.

that lies on the midplane in our example. It is now possible to determine the position of point $p$ on the shifted plane. The shifted point is

$$
\begin{aligned}
p_{2} & =\left[\begin{array}{l}
x_{2} \\
y_{2} \\
z_{2}
\end{array}\right] \\
& =p \pm \delta \bar{N} \\
& =\left[\begin{array}{c} 
\pm \frac{-C_{x} \delta}{\sqrt{C_{x}^{2}+1+C_{z}^{2}}} \\
C_{c} \pm \frac{\delta}{\sqrt{C_{x}^{2}+1+C_{z}^{2}}} \\
\pm \frac{-C_{z} \delta}{\sqrt{C_{x}^{2}+1+C_{z}^{2}}}
\end{array}\right]
\end{aligned}
$$

where $\delta$ is one half the phantom dimension, and the \pm determines positive or negative shift needed to specify both ideal planes resulting from the midplane. Inputting $p_{2}$ into the original plane expression, Equation 1, will make it possible to solve for the offset term for the shifted plane.

$$
\begin{aligned}
D_{\text {shifted }} & =N^{T} p_{2} \\
& =\left[\begin{array}{c}
-C_{x} \\
1 \\
-C_{z}
\end{array}\right]^{T}\left[\begin{array}{c}
\frac{-C_{x} \delta}{\sqrt{C_{x}^{2}+1+C_{z}^{2}}} \\
C_{c} \pm \frac{\delta}{\sqrt{C_{x}^{2}+1+C_{z}^{2}}} \\
\frac{-C_{z} \delta}{\sqrt{C_{x}^{2}+1+C_{z}^{2}}}
\end{array}\right] \\
= & \pm \frac{C_{x}^{2} \delta}{\sqrt{C_{x}^{2}+1+C_{z}^{2}}+C_{c} \pm \frac{\delta}{\sqrt{C_{x}^{2}+1+C_{z}^{2}}}} \\
& \pm \frac{C_{z}^{2} \delta}{\sqrt{C_{x}^{2}+1+C_{z}^{2}}} \\
= & C_{c} \pm \delta \sqrt{C_{x}^{2}+1+C_{z}^{2}} .
\end{aligned}
$$

Therefore, we see that the amount to shift the midplane is equal to $\delta \sqrt{C_{x}^{2}+1+C_{z}^{2}}$. The same method follows as well for the other planes.

\section{Distortion Correction Parameters}

The third and final stage involves performing the actual gradient nonlinearity distortion correction. The distortion correction model is based on the sum of spherical harmonics. Using the ideal planes obtained from the previous stage, theoretical "undistorted" data points will be calculated using the ideal plane equations and acquired data points. The undistorted data points are then used to calculate the coefficients of the distortion correction model. Three sets of coefficients are necessary, one for each dimension. Once the model is known it can be applied to all studies performed on the particular scanner.

In particular, let $x_{i}, y_{i}$, and $z_{i}$ be the distorted coordinates of some point $i$ measured by an MR scanner. Further let $\bar{x}_{i}, \bar{y}_{i}$, and $\bar{z}_{i}$ be the undistorted coordinates that corresponds to $x_{i}, y_{i}$, and $z_{i}$, that is $\left(\bar{x}_{i}, \bar{y}_{i}, \bar{z}_{i}\right)$ is the point on the ideal plane that produced the point $\left(x_{i}, y_{i}, z_{i}\right)$ in the MR image. Taking the first spherical harmonics, the correction model takes the form

$$
\begin{gathered}
\bar{x}_{i}=x_{i}\left(1+K_{x_{0}}\left(x_{i}^{2}+y_{i}^{2}\right)+K_{x_{1}} z_{i}^{2}+K_{x_{2}}\left(x_{i}^{2}+y_{i}^{2}\right)^{2}\right. \\
\left.\quad+K_{x_{3}} z_{i}^{2}\left(x_{i}^{2}+y_{i}^{2}\right)+K_{x_{4}} z_{i}^{4}\right), \\
\bar{y}_{i}=y_{i}\left(1+K_{y_{0}}\left(x_{i}^{2}+y_{i}^{2}\right)+K_{y_{1}} z_{i}^{2}+K_{y_{2}}\left(x_{i}^{2}+y_{i}^{2}\right)^{2}\right. \\
\left.\quad+K_{y_{3}} z_{i}^{2}\left(x_{i}^{2}+y_{i}^{2}\right)+K_{y_{4}} z_{i}^{4}\right), \\
\bar{z}_{i}=z_{i}\left(1+K_{z_{0}}\left(x_{i}^{2}+y_{i}^{2}\right)+K_{z_{1}} z_{i}^{2}+K_{z_{2}}\left(x_{i}^{2}+y_{i}^{2}\right)^{2}\right. \\
\left.\quad+K_{z_{3}} z_{i}^{2}\left(x_{i}^{2}+y_{i}^{2}\right)+K_{z_{4}} z_{i}^{4}\right) .
\end{gathered}
$$

Note that we know everything in these equations except the $K_{x_{j}}, K_{y_{j}}$, and $K_{z_{j}}$ terms, which are the desired correction parameters. We solve for the $K_{x_{j}}$ using the ideal planes whose normal is in the $x$ direction, i.e. the YZ planes, and similarly for the $K_{y_{j}}$ and $K_{z_{j}}$ terms. Since we started our 
example for the top and bottom edges of the images in an axial study results, i.e. a plane oriented in XZ, we will show the solution for this case. The other cases follow directly.

Begin by noting that $\bar{y}_{i}$ can be calculated using the ideal plane using the fact that since the distortion for this face is essentially all in the $\mathrm{Y}$ direction, we can assume the ideal $\mathrm{X}$ and $\mathrm{Z}$ coordinates $\left(\bar{x}_{i}\right.$ and $\left.\bar{z}_{i}\right)$ will be the same as the distorted $\left(x_{i}\right.$ and $\left.z_{i}\right)$.

$$
\begin{aligned}
C_{c} \pm \delta \sqrt{C_{x}^{2}+1+C_{z}^{2}} & =N^{T} p_{\text {ideal }} \\
& =\left[\begin{array}{c}
-C_{x} \\
1 \\
-C_{z}
\end{array}\right]^{T}\left[\begin{array}{c}
x_{i} \\
\bar{y}_{i} \\
z_{i}
\end{array}\right] \\
C_{c} \pm \delta \sqrt{C_{x}^{2}+1+C_{z}^{2}}+C_{x} x_{i}+C_{z} z_{i} & =\bar{y}_{i} .
\end{aligned}
$$

Knowing the ideal point $\left(x_{i}, \bar{y}_{i}, z_{i}\right)$ and the distorted points $\left(x_{i}, y_{i}, z_{i}\right)$, we substitute into Equation 14 and note that it is linear in the $K$ terms. Using all $n$ points of both planes oriented in the $\mathrm{XZ}$ direction we find

$$
\begin{aligned}
& S=T K_{y} \\
& S=\left[\begin{array}{c}
S_{0} \\
S_{1} \\
\vdots \\
S_{2 n-1}
\end{array}\right] \\
& S_{i}=\bar{y}_{i}-y_{i} \\
& T=\left[\begin{array}{ccccc}
T_{0,0} & T_{0,1} & T_{0,2} & T_{0,3} & T_{0,4} \\
T_{1,0} & T_{1,1} & T_{1,2} & T_{1,3} & T_{1,4} \\
\vdots & \vdots & \vdots & \vdots & \vdots \\
T_{2 n-1,0} & T_{2 n-1,1} & T_{2 n-1,2} & T_{2 n-1,3} & T_{2 n-1,4}
\end{array}\right] \\
& T_{i, 0}=y_{i}\left(x_{i}^{2}+y_{i}^{2}\right) \\
& T_{i, 1}=y_{i} z_{i}^{2} \\
& T_{i, 2}=y_{i}\left(x_{i}^{2}+y_{i}^{2}\right)^{2} \\
& T_{i, 3}=y_{i}\left(x_{i}^{2}+y_{i}^{2}\right) z_{i}^{2} \\
& T_{i, 4}=y_{1} z_{1}^{4} \\
& K_{y}=\left[\begin{array}{l}
K_{y_{0}} \\
K_{y_{1}} \\
K_{y_{2}} \\
K_{y_{3}} \\
K_{y_{4}}
\end{array}\right]
\end{aligned}
$$

Using a least squares solution technique, such as $\mathrm{QR}$ or SVD, allows us to solve for $K_{y}$. This process is repeated for the other correction parameters $K_{x}$ and $K_{z}$. Once we have the correction parameters we can take any MR image from the same scanner and use Equations 13-15 to solve for the undistorted points $\left(\bar{x}_{i}, \bar{y}_{i}, \bar{z}_{i}\right)$ given the distorted image points $\left(x_{i}, y_{i}, z_{i}\right)$.

\section{Results and Conclusions}

Initial testing has only been done on the phantom's faces, which when corrected returns the ideal planes to an accuracy of $.12 \mathrm{~mm}$ to $.14 \mathrm{~mm}$ on four of the six sides, which is about one third of a pixel, see Figure 4. While it is not surprising that correcting the image that was used to find the correction parameters would do well, it is surprising that it does so much better than half a pixel. The two sides which did not have an accuracy around $.13 \mathrm{~mm}$ were both the $\mathrm{x}$ planes, and they were only accurate to $.25 \mathrm{~mm}$. On investigating we discovered that the $\mathrm{x}$-gradient coil of the scanner was misaligned, which caused an asymmetry in the distorted faces violating our assumption of gradient field symmetry with respect to the midplane. When corrected we expect all the numbers will improve, but particulary the accuracy of the x planes.

We are acquiring a phantom with different geometry that we can use to validate the correction. The performance of the distortion correction algorithm will be tested by targeting markers with known coordinates in the validation phantom. The accuracy of the distortion correction can then be determined, and any necessary adjustments can be applied to achieve the goal of submillimeter accuracy. The MR distortion correction technique is also being planned to be used as part of a preclinical study of the new functional radiosurgery system, which will be tested on rats.

A correction technique for MR images is introduced, which can be found in a straightforward manner. The technique involves the calculation of six least squares problems, using any reasonable technique desired. Furthermore, once the parameters are found the use of the technique does not involve inversion of a non-linear function as other techniques do; rather it only involves calculating a small multivariable polynomial. Initial results are very promising and further testing is planned.

\section{References}

[1] G. Bednarz, M. Downes, B. Corn, W. Curran, and H. Goldman. Evaluation of the Spatial Accuracy of Magnetic Resonance Imaging-Based Stereotactic Target Localization for Gamma Knife Radiosurgery of Functional Disorders. Neurosurgery, 45:1156-1161, 1999.

[2] S. Doran, L. Charles-Edwards, S. Reinsberg, and M. L. MO. A Complete Distortion Correction for MR Images: I. Gradient Warp Correction. Phys. Med. Biol., 50:1343-1361, 2005.

[3] D. Kondziolka. Functional Radiosurgery. Neurosurgery, 44:12-20, 1999.

[4] S. Langlois, M. Desvignes, J. Constans, and M. Revenu. MRI Geometric Distortion: a Simple Approach to Correcting the Effects of Non-Linear Gradient Fields. J. Magn. Reson. Imaging, 9:821-831, 1999. 
[5] T. Lee, K. Schubert, and R. Schulte. Software Development For Correction of Gradient-Nonlinearity Distortions in MR Images. In P. P. Dey, editor, Proceedings of ICCSA 2006, 2006.

[6] D. Wang, W. Strugnell, G. Cowin, D. Doddrell, and R. Slaughter. Geometric Distortion in Clinical MRI Systems Part I: Evaluation Using a 3D Phantom. J. Magn. Reson. Imaging, 22:1211-1221, 2004.

[7] D. Wang, W. Strugnell, G. Cowin, D. Doddrell, and R. Slaughter. Geometric Distortion in Clinical MRI Systems Part II: Correction Using a 3D Phantom. J. Magn. Reson. Imaging, 22:1223-1232, 2004.

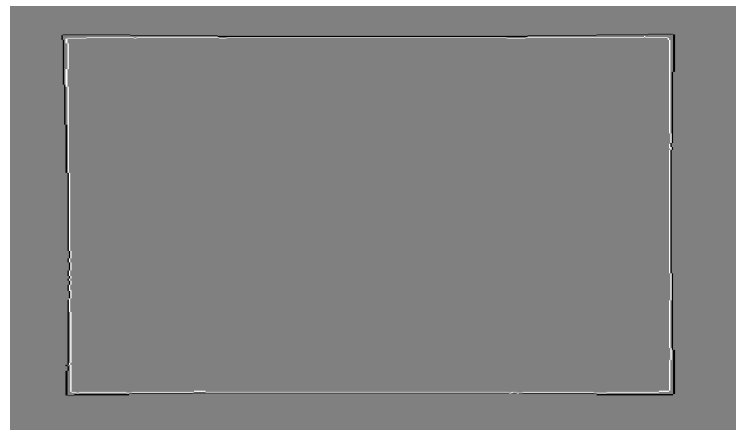

(a) axial slice

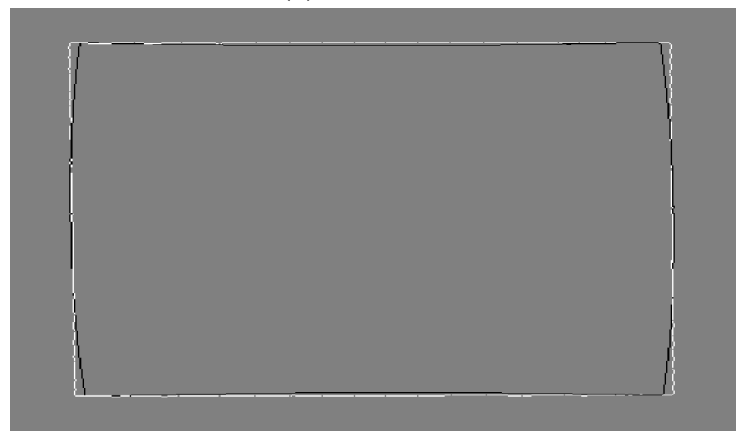

(b) coronal slice

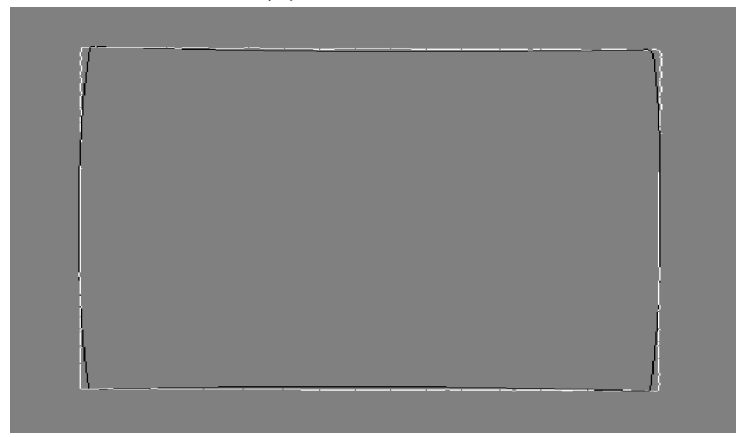

(c) sagittal slice

Figure 4. Sample distorted (black) and corrected (white) phantom image slices from (a) axial, (b) coronal, and (c) sagittal sequences. 